\title{
Information Extraction from Text using Text Mining
}

\author{
Shabanaunnisa Begum
}

\begin{abstract}
We're living in an technology of extended strain and intellectual problems. The extended diploma of strain \& force outcome in preference of to the form of people showing suicidal inclinations and therefore a larger shape of peoples are commit suicides pressure may be brought on able to family argument, task un satisfaction, healths troubles, and masses of others. inside the worldwide of modem computing, human beings feel loose to percentage their view and emotions over societal media for friends and family member via service together with text. to the kept nature and busy schedule of citizens it is pretty not easy to have interaction with pals and own relations people in individual, consequently community media structures are taken into consideration because of to the truth the maximum utilize platform for conversation. The purpose of this paper an estimate the suicidal incidents of a person thru using records mining technique to the textual content text somebody send to related humans. thru way of analyzing the additives of to the textual content messages we are capable of estimate the suicidal dispositions of a person without a doubt so important steps may be taken that allows you to keep the life of to the priority in this paper $i$ referred to about text mining, tokenizations finding, emoji conversion, feeling assessment, estimation mining, KNNalgorithms.
\end{abstract}

Key terms: textual content mining, facts discovery, feeling evaluation, estimation mining.

\section{INTRODUCTION}

The needs for the use of evaluation strategies to text message arrive with the ever growing suicides fees in severa components of to the planet. save the being of human is that the mission of excessive significance for a country. on the manner to keep away from dropping the lifestyles of dad and mom, their sentiments need to be an extended manner-famed and incidental actually so the popular steps may be in use on time. the remarkable manner to recognize the emotion of a persons is with the useful resource of to the use of records processing technique to the text communication a person sends. If a person signs code of hyper strains inform the mother and father about to person will facilitate in save the existence as a trouble.

Text device is finished at the textual content obtained from the patron. text pre device consists of tokenization, save youphrase-elimination and stemming and some opportunity strategies.

To kenization includes rending the textual content in the shape of phrases referred to as tokens. Tokenization is employed to identify key phrases within the flow into of texts. prevent-phrase-removal is that the technique of elimination of phrases that don't deliver a completely specific because of this within the document just like the, and, this ... and masses of others. Stemming is finished to get the idea phrase of to the data and do away with, and so on.This paper specializes in emotion evaluation for predict the pressure degree of an individual. The calculation version includes of SVM \& proper enough-NNalgorithms. that is frequently finished via feed the gadget with a information set for preparation the device. This system may be completed in absolutely outstanding conditions concerning opportunity domains. This approach can be accustomed are searching out the outcomes of elections as fast as finished at large scale and for a couple of subject. it is surprisingly powerful in predict the outcome concerning in reality super opinion of humans. it may be accustomed get preceding records regarding terror assaults or unorganize violent protest . Emoticon vicinity unit a virtually crucial a element of any do not forget range language above the web. it is moreover outstanding- stated will be the mainly communicative piece of any textual content communication as they create about the $\$ 64000$ spirit of to the language the numerous contrary numbers. in the long run, it is of pinnacle significance to have a take a look at the emoticons applied in any textual content message in order that the $\$ 64000$ sentiment of to the text is offered.

\section{PROPOSED METHOD}

The projected method enables to avoid losing life of folks that probable undergoing troubles of hyper stress or the alternative hassle in an attempt to revealknowledge deadly to them. The purpose is to remove statistics with the text communication of to the consumer and utilize for severa abilties like sentiments assessment. The version conjointly includes the assessment of emoticons an extraordinary manner to truly observe the statement.

\section{Records set description}

The records is obtain by means of way of to the usage of extract all the textual content communication send through hassle. This could be executed with many belongings like fb, Whatsapp, and many others. all the messages deliver thru the ones digital conversation services square measure maintain on for the duration of a information anywhere we're able to observe our version and observe the feelings. |the data|the statistics\} set can incorporate textual content type of records and emoticons. No unique form of records like images are going to be analyze during the model. 


\section{Information Extraction from Text using Text Mining}

\section{MODEL FACTORS}

\section{A. Sentiment assessment}

An element statistics the statistics the information allotted a

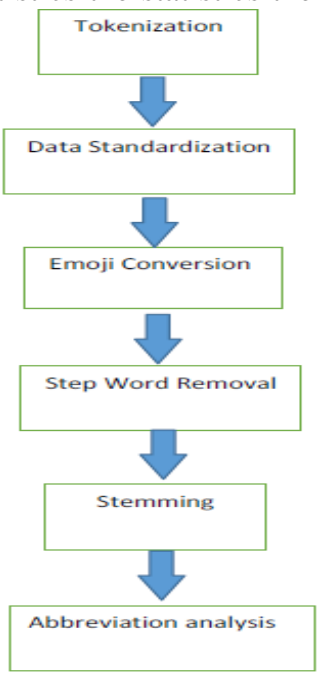

Figure 3. Steps in Text Message Processing

sentiment like high satisfactory or terrible and moreover the amount of it through the usage of way of playacting records pre-processing victimization SVM algorithmic application.

\section{B. Textual content Pre-processing}

The strategies concerned in textual content pre-processing rectangular measure. Tokenization: each new message is break up into sensible phrases called tokens.

\section{Statistics consistency}

It includes convert all phrases inside the communication in everyday type, convert all phrases in grapheme. instance. "The marketplace is close to John residence" is born-once more to "the marketplace is close to john's house".

Emojis conversions: The emoticon present in to the textual content messages are assign a key-phrase primarily based definitely on to the appearance they invent.

The emoticons are categorized into following instructions: First rate emoticon: the ones are the emoticon which deliver tremendous reaction and are modified by means of high first-rate phrases based totally at the photo.

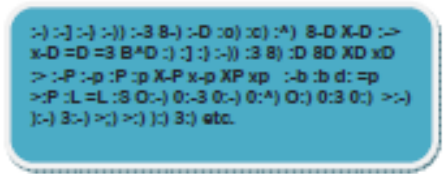

Figure 1. Positive Emoticons

Terrible emoticons: those emoticon replicate the unhappy or concerned sentiments of to the challenge and are consequently changed by poor terms.

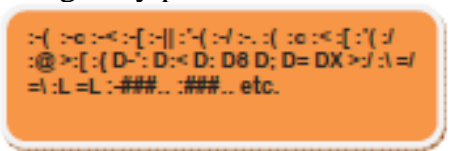

Figure 2. Negative Emoticons

End-phrase-elimination: The terms within the text that don't carry a completely unique which means that square degree eliminated form of a, the, then, and so on.

Stemming: It includes getting the concept word just like every statement through falling suffixes ion, and so on.

Abbreviation assessment: commutation the abbreviation right now in to the message via their full forms. Ex:fb through fb weight unit with the beneficial useful resource of greeting, and so on.

- N-gram

The subsequent step as rapid as statistics pre-dealing out is $\mathrm{N}$ gram options removal. Ngram can be a sequence of $\mathrm{n}$ tokens. Ngram may be a form exceptionally huge executed in statistics processing responsibilities. The version create Ngrams with messages at the knowledge set to remove keywords options with the information set.

For $\mathrm{n}=$ three a series of 3 -words to every communication is generate. method of Ngram will increase the potency \& accurateness of to the classification step attributable to the feature extract from 3 sequence of token combination. Example. "What is your name" is analysed as "what is your" "is your name".

\section{Term Frequency}

The sort of instances a token occurs in every records styles is known as its term frequency. phrases having immoderate frequency have better courting with the patterns.

\section{Inverse report Frequency}

Idf issue is employed to decrease the load of words that occur fairly often in the facts set and to extend the burden of phrases that occur seldom.

\section{SUPPORT Vector MAChines}

An ensuing flow of words when the text pre- process steps is processed by SVM formula so as to classify the communication as "usual" or "critical"emotion. the method is apply on each message in information set so as to classify the chat jointly among "normal" and "critical" sentiment. Therefore we'll get a sentiment related to the messages related to the user. SVM's square measure supervised learning models that square measure used for classification and multivariate analysis of knowledge

Utilize. A SVM form constitute examples as point in residence, completely special categories of examples rectangular degree divided through an specific gap that must be as extensive as ability. New examples once mapped into the house square measure foreseen to belong to a category of examples supported that element of to the gap they fall.

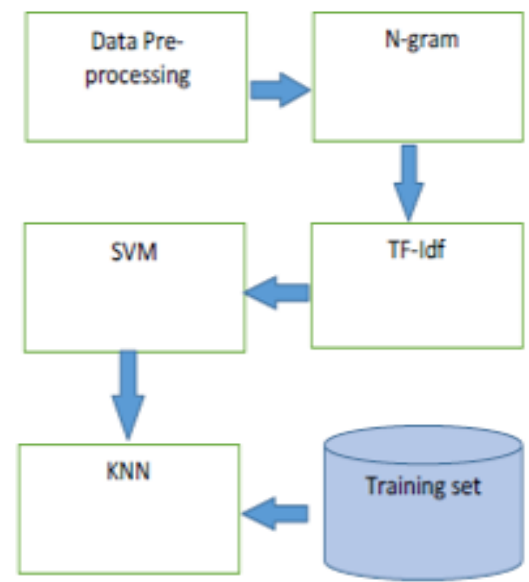

Fig4. Differnet steps in Data Proessess and Analysis 


\section{KNN ALGORITHMIC PROGRAM}

The result obtain from assist Vector Machines algorithmic software program region unit clusters of 2 emotions for class label "usual" and "important". supported the output KNNalgorithmic application is carried out a good manner to deduce the feelings of to the difficulty. The input for KNN algorithmic program is that the feelings coupled with all the chats challenge is concerned in. The closing step is to expect the response of to the person supported the gathered function set. data is cut up into schooling and trying out sets, and KNN algorithmic software program is hired to are looking forward to the sentiment. KNN algorithmic application may be a methodology for classifying statistics supported the near schooling sets in to the aspect vicinity. label is appointed identical because of to the truth the closestK times at the education set. KNNmay be a sort of lazys learner method. KNN algorithmic software program is considered a versatile and easy type approach supported device gaining knowledge of thoughts.

\section{RESUlt ANALYSIS}

The stop output obtain as the planned form gives the calculable sentiment prediction of to the concern supported the text messages sent via using the client. the following output are frequently hired in severa topics, the intellectual troubles and pressure degree is calculable and for that reason virtually in case of "critical" sentiments the friends and members of to the circle of relatives of to the subject will take movements to encourage, inspire and uplift the emotional stature of to the mission so fundamental to the concord and peace of mind of to the problem. so such sentiment evaluation fashions ar a call for for shaping the society into an prevalence location.

\section{FUTURE SCOPE}

The deliberate version are often employed in matters anywhere sentiment assessment is needed to attain the desired end end result and use it for severa without a doubt remarkable capabilities like critic evaluations for inns, films, films, and plenty of others. Sentiment evaluation strategies until presently are wont to sight the polarity inside the mind and evaluations of all of to the customers that get proper of entry to social media. companies ar as a substitute concerned to realise the thoughts of human beings and the manner they may be responding to any or all of to the products and services spherical them. Businesses use sentiment assessment to evaluate their merchandising campaigns and to enhance their merchandise. Organizations cause to use such sentiment evaluation device inside the regions of customer feedback, advertising, CRM, and e-trade.

\section{CONCLUSION}

The planned version takes input from the facts set created with the aid of using amassing all of to the textual content messages deliver by the use of to the state of affairs. all of to the messages is likewise from without a doubt considered considered one of a kind social media structures like $\mathrm{fb}$, whatsapp, and masses of others. The messages ar then preprocessed to get the key phrases from the records gadgets. as soon as preprocessing we have a propensity to use probabilistic language models like $n$
Gram. Associate weights to the facts set mistreatment TF-Idf will boom ordinary potency of classify algorithms. destiny steps is to apply the classify algorithms to categorise the conversation "normal" or "important" preliminary a supervise set of policies is employed this is SVM as it prove to be quite for like computation and so partner degree unattended algorithmic software program application is employed that successively will increase the performance substantially, in our case we have a tendency to apply the $\mathrm{KNN}$ algorithmic application. so we will be inclined to recommend to deliver a considerably for your rate variety approach of locating the response of to the person via analyzing the text messages and conjointly gadget emoticons. Emoticons ar quite commonplace tokens in any text message within the new worldwide, so we've got were given were given a tendency to want to conjointly target inner your fee range tactics in which to examine them. we have were given regenerate emoticons to take into account kind for our computation approaches. so this model may be a name for and a life saviour inside the these days.

\section{REFERENCES}

1. K. Tan, Steinbach, Introduction to Data Mining, 2006.

2. C. Paper, Preprocessing techniques for text mining preprocessing techniques for text mining, J. Emerg. Technol. Web Intell., 2016.

3. D. Lyon and B. Cedex, N-grams based feature selection and text representation for Chinese Text Classification Zhihua WEI, Int. J. Comput. Intell. Syst., 2(4), 365-374, 2009.

4. J.C.B. Christopher, A tutorial on support vector machines for pattern recognition, Data Mining and Knowledge Discovery, 2(2)(1998), 121-167.

5. E.-H. Sam Han, G. Karypis and V. Kumar, Text Categorization Using Weight Adjusted k-nearest Neighbor Classification, Springer, 2001.

6. J. Han, M. Kamber and J. Pei, Data Mining: Concepts and Techniques, Morgan Kaufmann, 2006.

7. D.A. Hull et al., Stemming algorithms: A case study for detailed evaluation, JASIS, 47(1)(1996), 70-84.

8. H. Isozaki and H. Kazawa, Efficient support vector classifiers for named entity recognition, in Proceedings of to the 19th international conference on Computational linguistics, Vol. 1, Association for Computational Linguistics, 2002.

9. M. James, Classification Algorithms, Wiley- Interscience, 1985.

10. T. Joachims, Text Categorization With Support Vector Machines: Learning With Many Relevant Features, Springer, 1998.

11. M. Kantardzic, Data Mining: Concepts, Models, Methods and Algorithms, John Wiley \& Sons, 2011.

12. L.S. Larkey and W.B. Croft, Combining classifiers in text categorization, in Proceedings of to the 19th annual international ACM SIGIR conference on Research and development in information retrieval, ACM, (1996), 289-297.

13. E.D. Liddy, Natural Language Processing, 2001. 